\section{PSICOLOGIA IBEROAMERICANA}

\section{Psicología lberoamericana}

ISSN: 1405-0943

psicología.iberoamericana@uia.mx

Universidad Iberoamericana, Ciudad de

México

México

Baile Ayensa, José I.; González Calderón, María J.

Trastornos de la Conducta Alimentaria antes del Siglo XX

Psicología Iberoamericana, vol. 18, núm. 2, julio-diciembre, 2010, pp. 19-26

Universidad Iberoamericana, Ciudad de México

Distrito Federal, México

Disponible en: http://www.redalyc.org/articulo.oa?id=133915921003

Cómo citar el artículo

Número completo

- Más información del artículo

Página de la revista en redalyc.org

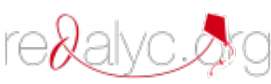

Sistema de Información Científica

Red de Revistas Científicas de América Latina, el Caribe, España y Portugal

Proyecto académico sin fines de lucro, desarrollado bajo la iniciativa de acceso abierto 


\title{
Trastornos de la Conducta Alimentaria antes del Siglo XX
}

\author{
Eating Disorders before the 20th Century
}

José I. Baile Ayensa

María J. González Calderón*

Facultad de Psicología

Universidad a Distancia de Madrid (UDIMA), España

\section{RESUMEN}

Los trastornos de la conducta alimentaria (TCAs), como la anorexia nerviosa y la bulimia nerviosa, han sido un problema de salud de gran prevalencia a finales del siglo XX y comienzos del XXI. Dichos comportamientos atípicos fueron inicialmente descritos como tales en el siglo XIX, si bien adquirieron entidad psicopatológica a partir de la década de los sesenta, considerándose trastornos "modernos" asociados a un excesivo culto al cuerpo y deificación de la delgadez, características de nuestra sociedad actual. No obstante, dichos problemas de salud podrían estar ocurriendo desde hace siglos. Este artículo revisa algunos de los casos más famosos, valorando las limitaciones de la sesgada información disponible, analizando similitudes y diferencias entre los supuestos casos históricos, y las manifestaciones actuales. El análisis histórico, en el que se constata la presencia de comportamientos alterados a lo largo de los últimos siglos, permite avanzar en los conocimientos actuales de los TCAs tanto desde el punto de vista definitorio como terapéutico.

Descriptores: Anorexia, bulimia, perspectiva histórica.

\section{ABSTRACT}

Eating Disorders (ED) such as Anorexia Nervosa and Bulimia Nervosa have been prevalent health problems at the end of the 20th century and the begining of the 21st. These disturbed behaviours were initially described as such in the 19th century although they didn't become a psychopathological entity till the 60s, when they were considered as "modern" disorders linked to an excessive body cult and slimness stress, features which characterize our current society. However, these health problems could have been happening for centuries. This paper reviews some of the most famous case studies, taking into account the limitations of the biased available data, analyzing the differences and similarities found between the historical cases studied and the current symptoms of both disorders. It was also considered whether the historical analysis carried out allows us to extend our current knowledge on the issue from both perspectives defining as well as therapeutic.

Key words: Anorexia Nervosa, Bulimia Nervosa, Historical approach.

\footnotetext{
* José I. Baile Ayensa y María J. González Calderón. Departamento de Psicología. Facultad de Ciencias Sociales y Jurídicas. Universidad a Distancia de Madrid (UDIMA). Camino de la Fonda, 20. C.P. 28400- Collado-Villalba (Madrid). España. Tfno: 918561699 // Fax 918561697. Correo electrónico para ambos autores: ignaciobaile@hotmail.com
} 


\section{INTRODUCCIÓN}

Desde hace algunas décadas, la sociedad occidental y la comunidad científica se han enfrentado a la proliferación de una serie de trastornos de salud, caracterizados por la alteración del comportamiento alimentario. Con un gran impacto en los medios de comunicación, la anorexia nerviosa y la bulimia nerviosa se han convertido en problemas enormemente conocidos; se cifra su prevalencia en torno al 0,5 al $1 \%$ en el caso de la primera y al 1 al $3 \%$ en el caso de la segunda en mujeres jóvenes occidentales (Baile, 2004).

Estos trastornos del comportamiento alimentario (TCAs) se clasifican en la actualidad, y siguiendo el Manual de Diagnóstico de la APA (2009) en tres categorías: la anorexia nerviosa, la bulimia nerviosa y los trastornos del comportamiento alimentario no especificado -(TCANE. Para la próxima edición del manual), la APA propone una nueva figura, la del trastorno por atracón (2010). Considerando los criterios diagnósticos, estos trastornos comparten un deseo de la persona de estar delgada (o miedo a engordar) que se articula en ciertos comportamientos alterados más específicos de cada trastorno; en el caso de la anorexia la restricción alimentaria, en el caso de la bulimia el descontrol del comportamiento, con fases restrictivas y compulsivas alternativamente.

La justificación del incremento espectacular de la prevalencia de los TCAs en la segunda mitad del siglo $\mathrm{XX}$ y en el comienzo del siguiente, así como su circunscripción a cierta cultura, la occidental, se ha asociado a ciertos factores predisponentes de carácter sociocultural (Levine \& Smolak, 2000) y, en especial, al efecto de ciertos valores sociales como la deificación de la delgadez en la mujer como modelo de belleza, y su imposición como canon a seguir a través de los medios de comunicación, todo ello impulsado por una potente industria de la belleza (cosméticos, productos dietéticos, cirugía, etcétera). La importancia de dichos factores socioculturales es tal, que algunos autores afirman que "puede asegurarse que sin su existencia, no existiría tampoco la anorexia nerviosa" (Toro \& Vilardell, 1987, p. 106).

Pero, ¿son los TCAs una manifestación específica de la sociedad occidental de los siglos XX y XXI como consecuencia de cierto contexto social patologizante?, ¿o quizá son trastornos consustanciales al ser huma- no, pero ahora ha repuntado su prevalencia? Algunos autores defienden que las alteraciones del comportamiento alimentario "en general" siempre han estado presentes pero que desde el siglo XIX tenemos específicamente los trastornos psicopatológicos concretos que hoy conocemos, debido a las variables socioculturales específicas de estos últimos siglos (Vandereycken, 2002). En los próximos apartados se realiza un breve análisis desde una perspectiva histórica y se valora qué nos puede aportar el mismo, desde el punto de vista explicativo y terapéutico.

\section{MÉTODO}

Para esta revisión se han consultado diversas fuentes bibliográficas directas (como la biografía de Santa Catalina de Siena escrita en el siglo XIV) e indirectas, como las publicaciones históricas, desde una perspectiva psicológica, que han tratado este tema, de las cuales se han seleccionado 10 para su inclusión en el estudio histórico.

Tras incorporar algunos de los datos clave de la revisión se procede a realizar su análisis crítico, así como sus posibles implicaciones actuales de la misma.

\section{RESULTADOS}

\section{Revisión de casos históricos con posible TCA}

A lo largo de la historia se tiene conocimiento de numerosos casos de personas con algún tipo de trastorno de conducta alimentaria, de los cuales los ejemplos de religiosas medievales y renacentistas son los más famosos, pero no los únicos. Utilizar el "control" del comportamiento alimentario como una forma de manifestación religiosa o ideológica ha sido común a numerosas culturas a lo largo de toda la historia, en algunos casos promoviendo y valorando la obesidad; en otros, el ayuno. Por ejemplo, Hinojosa (2009, p. 184) recuerda estas palabras de Santa Teresa de Jesús cuando fundó el convento de San José en Ávila: "Y no penséis, hermanas mías, que por eso os ha de faltar de comer, yo os aseguro (...) Si haciendo vosotras esto muriéreis de hambre, ¡bienaventuradas las monjas de San José! Aquí os digo yo serán aceptas vuestras oraciones, y haremos algo de lo que pretendemos. Esto no se os olvide, hijas mías, por amor del Señor; pues dejáis la renta, dejad el cuidado de la comida; si no, todo va perdido". 
Se han recopilado multitud de posibles casos de TCA en el pasado. Así, Bell (1985) menciona hasta 261 casos de mujeres religiosas italianas desde el siglo XIII, y Parry-Jones \& Parry-Jones (1994) cuentan hasta 360 casos de ayuno voluntario entre 1500 y 1939 . Muchos de éstos se refieren a personas famosas por su vinculación religiosa o política, o por la singularidad del caso que llegó a ser descrito. A modo de ejemplo, se indican algunas de las más famosas (Bell, 1985; Keel \& Klump 2003; Toro 1996):

- Siglos XIII al XVI: Santa Catalina de Siena, Santa Verónica, María de Oignies, Beatriz de Nazaret, Margarita de Yperen, Juliana de Lieja y Columba de Rieti.

- Siglos XVI al XIX: Jane Grey (Reina Inglaterra), Jeanne Balan y Marthe Taylor.

- Siglo XIX: Anne Moore, Margaretha Weiss, Barbara Kremers y Eva Vliegen.

Los casos descritos con anterioridad resultan de especial interés al tratarse de ejemplos extremadamente ilustrativos, de los cuales se tiene información fehaciente y que son clave para poder realizar el análisis histórico objeto del presente estudio.

En el siglo XIX existen numerosos y floridos casos de "chicas ayunadoras" (Brumberg, 1989), y en una naciente ciencia psicopatológica se crea el oportuno caldo de cultivo para su clasificación y catalogación; así, dos famosos psiquiatras, Lasëgue (1873) y Gull (1888), elaboran de manera independiente una primera definición del trastorno, que viene a denominarse "anorexia histérica" o "anorexia mirabilis" (Vanderycken \& Van Deth, 1990).

A efectos ilustrativos se realiza un breve análisis de dos de los casos más famosos: Santa Catalina de Siena (s. XIV) y Anne Moore (s. XIX).

Santa Catalina de Siena (1347-1380). Catalina de Siena es una de las figuras más importantes del santoral cristiano, y junto con Santa Teresa de Jesús y Santa Teresa de Lisieux comparte el título de doctoras de la Iglesia. Nació en 1347 en Siena, en el seno de una familia muy numerosa y tradicional. Al parecer, desde niña tuvo visiones místicas y decidió dedicarse a la vida contemplativa, lo que contradecía el deseo de sus padres de casarla tempranamente. Esto le generó graves problemas con su familia y, entre otras reacciones, decidió someter a su cuerpo a una serie de fuertes restricciones, entre ellos el ayuno. Se tienen descripciones de su comportamiento alimentario gracias a la obra biográfica de su confesor Raymundo de Capua, como la que aparece en el siguiente texto (Capua, 1947, pp. 24-25):

"Desde la infancia, Catalina apenas había probado la carne; ahora se la prohibió de la manera más absoluta y tanto se habituó a la privación de este alimento que terminó por no poder soportar el olor de él sin que su estómago se resintiese (...) A la edad de 15 años renunció por completo a él e ingería únicamente agua pura. Y restringiendo día por día la cantidad de los alimentos llegó a no comer más que un pedacito de pan y algunos vegetales sin cocer (...). Su existencia era un milagro, y los médicos que la reconocieron me manifestaron que el caso no tenía explicación científica. Durante todo el tiempo que tuve el privilegio de ser testigo de su vida no tomó alimento ni ingirió bebida en cantidad suficiente para sostenerla y sin embargo lo soportaba con alegría, aunque a costa de grandes sufrimientos y extraordinaria fatiga".

Según esta fuente biográfica, en el momento en que se niega a casarse, tenía unos 16 años y subsistía de pan, agua y vegetales crudos, vestía con ropas ligeras y un cinturón de hierro. Junto al ayuno también se imponía otras restricciones, por ejemplo, tenía periodos de varios años de silencio total, salvo con su confesor; o periodos sin sueño; se llega a relatar que era capaz de dormir treinta minutos cada dos días. Tras esta actitud tan autolesiva, consigue que le dejen dedicarse a la vida religiosa y entra en la orden dominicana. Aunque ya no tiene motivos para mantener su protesta, continúa con su alteración alimentaria, pues declara que ya no es capaz de ingerir alimento de forma normal.

Se conservan cartas y documentos de su confesor, en las que éste relata que Santa Catalina mantuvo el ayuno hasta su muerte, y que era habitual que vomitara lo que ingería porque su cuerpo no soportaba alimento alguno. Murió extremadamente delgada y enferma en 1380, a los 33 años de edad.

Anne Moore "The Fasting Woman of Tutbury" (La ayunadora de Tutbury. 1761-1813). Siguiendo a Toro (1996), uno de los ejemplos más famosos de tras- 
torno de la conducta alimentaria es el de Anne Moore. La singularidad del caso radica en ser uno de los primeros en los que se realizó un estudio médico riguroso de un comportamiento de ayuno tan extraordinario que había generado muchas dudas sobre su veracidad.

Anne, de posición humilde, inició su ayuno cuando trabajaba de sirvienta y tenía unos 27 años. Aunque inicialmente lo justificaba por la incapacidad de alimentarse debido a su trabajo con ulcerosos, dado que tenía que convivir permanentemente con imágenes de heridas, posteriormente adujo que eran motivos religiosos los que le hacían mantenerse en ayuno. Este componente se convirtió paralelamente en una fuente de ingresos, dado que numerosos "peregrinos" la visitaban.

El caso se hizo muy famoso ya que, al parecer, podía pasar muchos días sin comer nada en absoluto, llegando a afirmar que llevaba años sin probar bocado, o sólo se alimentaba de unas pocas pasas de Corinto. Dicha fama hizo que se creara una comisión investigadora por parte del Colegio Real de Médicos, con objeto de comprobar la veracidad de poder pasar tanto tiempo sin alimentarse. Se decidió realizar un estudio riguroso y, para ello, se pensó que lo mejor era encerrarla a solas en una habitación sin acceso a ningún alimento oculto y observar cómo sobrevivía. Conforme pasaba el tiempo, su salud empeoraba notablemente. Al permitir que su hija la visitara, se descubrió que ésta utilizaba diferentes tretas para darle alimento a su madre durante las visitas, como pasarle trozos de comida a través de besos.

Si bien se descubrió que era imposible sobrevivir sin alimento, no deja de tratarse de un caso de alteración del comportamiento alimentario, aunque la poca información al respecto, nos puede hacer dudar si constituyó un trastorno primario o secundario.

\section{SIMILITUDES Y SINGULARIDADES DE LOS CASOS HISTÓRICOS FRENTE AL DIAGNÓSTICO ACTUAL.}

Consideraciones previas Establecer, en el siglo XXI, si los ejemplos históricos son verdaderamente casos de TCA tal y como los entendemos hoy en día es muy difícil, básicamente por la información sesgada a la que tenemos acceso, así como por la aplicación de patrones diagnósticos de forma anacrónica. A continuación se señalan algunas de las consideraciones que se deben tener en cuenta:

- Las técnicas historiográficas informativas empleadas (biografías de exaltación, cartas con confesores o familiares, análisis de imágenes en arte, comentarios aislados de contemporáneos, etc.) son sesgadas y poco consistentes.

- Es difícil realizar los perfiles psicopatológicos de los casos históricos, ya que se emplean descripciones parciales, con información escasa y sesgada, exaltación de comportamientos y alteraciones por parte de los cronistas con fines religiosos y por la imposibilidad de descartar patologías de otro orden.

- En el pasado se omitían datos que no eran objeto de interés y que hoy en día consideramos importantes, por ejemplo, si existía miedo a engordar, se presentaba alteración del esquema corporal, o si tuvo lugar y en qué momento preciso se vio afectada la menstruación.

- Por último, habría que destacar que puede ser incorrecto realizar valoraciones psicopatológicas sobre comportamientos alterados en el pasado empleando criterios modernos, sobre todo en trastornos tan dependientes de la cultura como los TCAs.

\section{Similitudes con los casos actuales}

A pesar de las limitaciones previamente señaladas, el estudio de los casos históricos permite establecer ciertos paralelismos con los que tienen lugar en la actualidad. A continuación se presentan los más destacados.

- En todos se observa una actitud alterada hacia el comportamiento alimentario. En dichos casos provenía de una fuerte motivación religiosa, oposicionismo a la familia o experiencias negativas de diversa índole. Sea cual fuere dicha razón, en todos los casos se asocia el trastorno con la alteración del comportamiento alimentario.

- Dicha alteración del comportamiento, tanto en el pasado como en la actualidad, se concreta en ciertas acciones similares, entre otras, el ayuno, la alimentación selectiva, el purgamiento o la alimentación errática, etcétera. 
- Al parecer, también podrían compartirse en la mayoría de los casos ciertos rasgos de personalidad, como son el perfeccionismo, la hiperactividad o la tendencia obsesiva-compulsiva. Capua (1947, p. 180) relataba lo siguiente de Santa Catalina:

"Hasta entonces, debido al dolor de costado que sufría y a otras dolencias que nunca la abandonaban, no oía la santa misa hasta la hora de Tercia. Así lo hizo durante toda la cuaresma yendo todos los días a la iglesia de San Pedro. Asistía a la misa, oraba más dilatadamente que nunca y regresaba a casa a la hora de Vísperas. Quienes la veían tendida en el lecho, no creían que fuese capaz de levantarse de él. Sin embargo, al día siguiente, al amanecer, se levantaba, salía nuevamente de casa y se iba por la «Vía del Papa», entraba en la Minerva, seguía por el «Campo di Fiore» y llegaba a buen paso hasta San Pedro, recorriendo una distancia capaz de fatigar a una persona robusta y en perfecta salud".

- También existen ciertas similitudes demográficas. Tanto en el pasado como en la actualidad la mayoría de los casos que presentan la sintomatología analizada son mujeres, y el inicio del trastorno se sitúa en la adolescencia-juventud.

- Procede destacar una última similitud: en muchos casos parece existir una disociación entre la motivación al inicio del trastorno y que lo obliga a persistir. Normalmente la alteración inicia por alguna motivación específica (religiosa, ascética, oposicionista, estética) pero, tanto en el pasado como en la actualidad, transcurrido un tiempo, las razones para mantener los comportamientos suelen ser otras si tenemos en cuenta que, aun desapareciendo la motivación inicial, muchas de las afectadas manifiestan que "no saben o pueden dejar dichas conductas".

\section{Diferencias con los casos actuales}

Aunque varias son las similitudes, también existen importantes diferencias entre aquellos casos y los actuales. Éstas pueden tener un fundamento real o provenir de un análisis erróneo de la información disponible, que puede estar sesgada y limitada por las razones ya expuestas anteriormente. Algunas de estas aparentes distinciones parecen ser las siguientes:

- En la actualidad, los TCAs tienen como elemento singular utilizar el comportamiento alimentario con objeto de conseguir un fin específico: el de mantener una determinada imagen corporal, de modo que dicha conducta es un medio, no un fin en sí mismo. Sin embargo, en muchos casos históricos analizados, el ayuno per se era el fin; o sea, no se pretendía estar delgada, entre otros motivos porque éste no era un valor social.

- Hoy en día la mayoría de los casos de TCA se clasifican dentro de la bulimia nerviosa, TCANE o probablemente trastorno por atracón -(que ya se ha propuesto como nueva categoría para la próxima edición del DSM), mientras que los casos restrictivos puros son los menos frecuentes. Sin embargo, en los casos históricos, al parecer predominaban los ejemplos restrictivos frente a los purgativos. Aunque quizá aquí sea la falta de información lo que confunda el análisis. Por otro lado, en la actualidad es difícil establecer la prevalencia de las personas que sufren trastornos purgativos, dado que, como no manifiestan una delgadez extrema, muchas personas lo sufren de forma oculta y en aparente compatibilidad con una vida normal.

- Aunque hay noticias de algunos casos de gran actividad física en el pasado, como se ha visto anteriormente, no se sabe que ésta fuera una actitud generalizada, en contraste con el uso de estrategias actuales para favorecer la delgadez, no sólo la mencionada hiperactividad, sino también el uso de acciones cosméticas, masajes, etcétera; quizá, como se ha indicado en la primera diferencia temporal, esto se debe a que el objeto en sí mismo no era la búsqueda de la delgadez, sino el ayuno como tal.

- Por último, se puede destacar una diferencia en cuanto al motivo inicial del trastorno, que en el pasado sería fundamentalmente un "deseo ascético" frente a la "insatisfacción corporal" actual. Aunque podría considerarse que ambos motivos, aparentemente diferentes, son la manifestación de un mismo factor común, la "presión social 
para cumplir con un valor social imperante en cada momento histórico".

Por todo lo recogido en estas líneas, en puridad, no podríamos hacer un diagnóstico de anorexia o bulimia de los casos históricos, siguiendo los criterios actuales recogidos en el DSM-IV-TR (2009), bien por falta de información o por incumplimiento de criterios comprobables, como son el porcentaje de pérdida de peso corporal o la presencia de amenorrea. Por tanto, si quisiéramos poner alguna etiqueta diagnóstica a dichos casos, sería más oportuno considerarlos como TCANE en lugar de ejemplos de anorexia o bulimia nerviosas.

¿Qué puede aportar este análisis histórico a la comprensión o abordaje de los TCAs en la actualidad?

La revisión de los ejemplos de TCA en el pasado, además del interés puramente histórico, revisten una importancia exegética para entender los casos actuales. Para algunos autores, este análisis permite determinar la existencia de verdaderos casos de TCA en el pasado, como apuntan Toro (1996, p. 39): “... así pues, puede defenderse que, si no todos, la mayoría de los casos descritos en la literatura anterior al siglo XX, eran auténticos casos de anorexia nerviosa," y Beumont (1991, p. 14): "los pacientes que Gull y Lasègue describían en 1870 se parecen a los pacientes de anorexia nerviosa de 1990, más claramente que los pacientes de demencia precoz de Kraepelin, los esquizofrénicos de Bleuler o los histéricos de Freud se parecen a sus paralelos contemporáneos". Si aceptamos esta tesis, ello podría ayudarnos a comprender mejor la verdadera naturaleza psicopatológica del trastorno, al constatar que desde siempre ha habido casos de TCA. Ello nos permitiría descontextualizar la etiología, dado que en la actualidad damos extremada importancia al peso del factor sociocultural relacionado con la presión estética. Es decir, podríamos afirmar que el factor sociocultural no es crucial y que, quizá, otros factores lo son de forma prioritaria. Ello no supone negar el peso de los factores socioculturales, pues aún asumiendo que en el pasado la presión hacia la delgadez no existía, sí que tenían lugar otros factores facilitadores del ayuno, como pueden ser la extendidas ideas ascéticas y religiosas.
Si asumimos, por tanto, que en el pasado han existido los TCAs, con sus singularidades específicas, evidentemente esto reforzaría la posible sustentación psicobiológica de los mismos, vía predisposición innata o alteración psicobiológica en la pubertad. Es decir, siempre han existido personas, mayoritariamente mujeres, con predisposición a sufrir los síntomas analizados, los cuales, si no constituyen una tendencia específica de TCA, sí podrían ser una predisposición hacia un grupo de trastornos del espectro obsesivo-compulsivo (Candil, 2002), que en algunos casos se manifestaría en anorexia o bulimia, si el contexto histórico promociona el ayuno o la purga para obtener algún beneficio. Aquellas mujeres que inician un ayuno por cierta presión social (ascética o estética) y tienen predisposición a sufrir un trastorno del espectro obsesivo-compulsivo, parece que podrían terminar desarrollando algún TCA.

Por otra parte, el análisis histórico también nos permite comprobar que independientemente de la razón de inicio de la alteración, el motivo por el que ésta se mantiene en el tiempo es diferente, y que esta disociación apoyaría la explicación psicobiológica de la persistencia en el trastorno: la inanición mantenida en el tiempo, junto a las sensaciones de control que genera, impiden que un individuo supere el trastorno aunque lo desee. Esta valoración sería coherente con otras investigaciones de carácter etológico, donde se ha comprobado una relación morbosa entre inanición y actividad física (Baile \& Garrido, 1999; Morse et al., 1995).

En la figura 1 puede apreciarse una re-interpretación del modelo explicativo de los TCAs que incluye las matizaciones expuestas previamente. Según dicho modelo, estos trastornos se producen por ciertos factores predisponentes (género femenino, baja autoestima, presiones sociales, etc.), y por la acción de factores desencadenantes (una situación de ansiedad asociada al cuerpo o la imagen corporal, por ejemplo) dan lugar al inicio de las alteraciones comportamentales, las cuales se mantienen en el tiempo por efecto de ciertos factores mantenedores (como puede ser el refuerzo de la sensación de control o ciertas alteraciones psicobiológicas). En el esquema representado en la figura 1 es muy común en la explicación de trastornos psicológicos y de salud. Para una descripción detallada del 


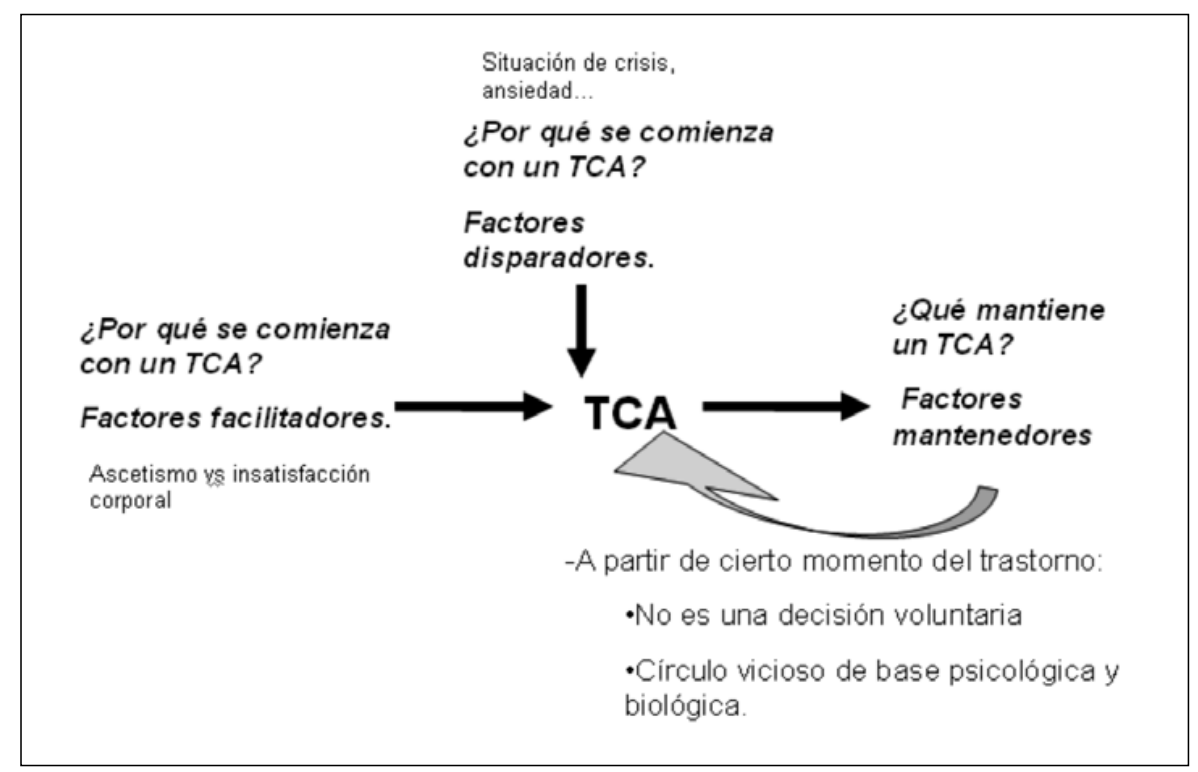

Figura 1. Modelo explicativo de los TCA, con aportaciones del análisis de los casos históricos modelo en el caso de los TCAs véase Toro \& Villardell (1987).

Considerar que existe una fundamentación psicobiológica por la cual las personas con TCA mantienen el trastorno por razones diferentes a una mera decisión voluntaria, tiene importantes implicaciones terapéuticas. Una de las estrategias clínicas clásicas es intentar convencer a las pacientes con TCA del problema que padecen, que lo asuman y empiecen a dejar de emitir las conductas patológicas asociadas, es decir, se pretende fundamentar la terapia en que el trastorno se inicia y se mantiene en todo momento por una decisión volitiva y revisable por el propio individuo. En este sentido, se aplican técnicas dialécticas, cognitivas o conductuales. Pero quizá a partir de un determinado momento del trastorno parece existir una alteración psicobiológica ocasionada por la inanición que "supera" la capacidad de decisión del paciente, y que debe conocer y manejar el terapeuta. Esta reflexión, por ejemplo, puede ayudar en un debate terapéutico actual sobre la importancia de trabajar prioritariamente unos objetivos $\mathrm{u}$ otros, $\mathrm{y}$ quizá la recuperación de cierto peso mínimo sea más importante de lo que se creía en un principio, no solo por motivos biomédicos, sino para facilitar la psicoterapia.

\section{CONCLUSIONES}

De la revisión llevada a cabo pueden extraerse las siguientes conclusiones:
- Los TCAs constituyen un grupo de alteraciones psicológicas que generan en la actualidad gran interés debido a su alta prevalencia entre las adolescentes y a la agresividad de su manifestación clínica. Se ha asumido que las tendencias estéticas actuales pueden ser la causa de la elevada extensión del problema.

- Sin embargo, se han constatado numerosos casos de chicas ayunadoras desde el siglo XIV, con perfiles aparentemente similares a los actuales TCAs. En relación a éstos, sería más oportuno hablar desde un punto de vista técnico de posibles casos de TCANE que de anorexia santa o anorexia $\mathrm{mi}$ rabilis, dado que la información disponible en la actualidad para poder hacer un diagnóstico preciso de las formas psicopatológicas más puras es muy sesgada.

- En muchos de estos casos históricos se conoce el motivo de inicio, normalmente ascético, si bien la causa del mantenimiento del comportamiento a lo largo del tiempo podría ser más compleja, independiente de decisiones volitivas $y$, probablemente, relacionada con una alteración psicobiológica resultado de la inanición, prueba de ello es que en varios casos se constató una incapacidad para abandonar el comportamiento alterado aunque se tuviera verdadera intención.

- Aunque la diferencia aparente más singular entre los casos de TCA en diferentes momentos 
históricos sea el motivo para iniciar el trastorno (ascetismo vs. insatisfacción corporal) quizá, si consideramos cuál es la base de ambas motivaciones, una presión social para considerar la delgadez y el ayuno como valor positivo, realmente no exista tal diferencia.

- La constatación de la existencia de comportamientos alimentarios alterados de fundamento psicológico a lo largo de la historia puede aportar información muy útil. Por una parte, desde el punto de vista del análisis etiológico: además de

\section{REFERENCIAS}

American Psychiatric Association (APA) (2009). Manual Diagnóstico y Estadístico de los Trastornos Mentales. DSM-IV-TR. Barcelona: Masson.

American Psychiatric Association (APA) (2010). DSM-5: The Future of Psychiatric Diagnosis. Disponible en: www.dsm5.org

Baile, J.I. (2004). Imagen corporal: perspectiva psicológica. México: ACD.

Baile, J.I. \& Garrido, E. (1999). Hiperactividad en anorexia nerviosa. Revisión. Revista Huarte de San Juan, 4-5, 149-159.

Bell, R.M. (1985). Holy anorexia. Chicago: University of Chicago Press.

Beumont, P.J.V. (1991). The history of eating and dieting disorders. Clinical Applied Nutrition, 1, 9-20.

Brumberg, J. (1989). Fasting girls; the history of anorexia nervosa. New York: Plume.

Candil, A.M. (2002). Una revisión del trastorno obsesivo en la infancia y en la adolescencia. Anales de Psiquiatría, 18, 342-348.

Capua,, R. de (1947). Vida de Santa Catalina de Siena. Buenos Aires: Espasa-Calpe (Original del s. XIV).

Gull, W.W. (1888). Anorexia Nervosa. Lancet, 1, 516-517.

Hinojosa, S. (2009). Santa anorexia. La noche oscura del cuerpo. Madrid: Maia Ediciones.

Keel, P.K. \& Klump, K.L. (2003). Are Eating Disorders Culture-Bound Syndromes? Implications for conceptualizing their etiology. Psychological Bulletin, 129, 747-769.

Lasëgue, Ch. (1873). De l'anorexie hystérique. Archives of General Medicine, 21, 385-403. la insatisfacción con la imagen corporal (que es la razón que actualmente se considera crucial) puede haber otras que promuevan y mantengan el ayuno, y que tengan más peso en estos trastornos, como son ciertos rasgos de personalidad. Pero también nos aporta información útil para encauzar el enfoque terapéutico para el abordaje actual de los TCAs, ya que se apoyaría la estrategia de no centrar la intervención tanto en las causas del inicio del trastorno, como en los mecanismos no volitivos de su mantenimiento.

Levine, M.P. \& Smolak, L. (2000). Los medios de comunicación y los disturbios de la alimentación: consecuencias en la prevención primaria. En: W. Vandereycken y G. Noordenbos, La prevención de los trastornos alimentarios (pp 39-79). Barcelona: Granica.

Morse A.D., Russell J.C., Hunt T.W., Wood G.O., Epling W.F. \& Pierce W.D. (1995). Diurnal variation of intensive running in food-deprived rats. Canadian Journal of Physiology and Pharmacology, 73, 15191523.

Parry-Jones, W. L. \& Parry-Jones, B. (1994). Implications of historical evidence for the classification of eating disorders. British Journal of Psychiatry, 165, 287-292.

Toro, J. (1996). El cuerpo como delito. Madrid: Ariel.

Toro, J. \& Vilardell, E. (1987). Anorexia Nerviosa. Barcelona: Martínez Roca.

Vanderycken, W. \& Van Deth, R. (1990). A tribute to Laségue description of anorexia nervosa (1873) with completion of its English translation. British Journal of Psychiatry, 157, 902-908.

Vanderycken, W. (2002). History of anorexia nervosa and bulimia nervosa. En: C.G. Fairbun y K. D. Brownell, Eating disorders and obesity. New York: The Guilford Press.
Fecha de recepción: Junio 2010 Fecha de publicación: Diciembre 2010 